American Journal of Nanotechnology 1 (2): 68-77, 2010

ISSN 1949-0216

(C) 2010 Science Publications

\title{
Effects of Adhesive on Coupling Efficiency of Planar Light Waveguide Packaging
}

\author{
${ }^{1,2}$ Yang Bo and ${ }^{1}$ Duan Ji-an \\ ${ }^{1}$ College of Mechanical and Electrical Engineering, \\ Central South University, Changsha 410083, P. R. China \\ ${ }^{2}$ Institute of Image Recognition and Computer Vision, \\ Hunan Normal University, Changsha 410081, P. R. China
}

\begin{abstract}
Problem statement: Advanced optoelectronic devices are a kind of key and basic components for next generation communication, untouched sensor, medical testing. They have lots of advantages including greater communication performance, larger capacity, and more fixable form. But light coupling and package structure seriously affects performance of optoelectronic devices. So a lot of attaching methods were proposed to package optoelectronic devices. A popular method which was widely used is a UV epoxy adhesive which can solidify the coupling interface with high mechanical strength and submicron displacement. In order to obtain high coupling efficiency, coupling approximation after adhesive is needed. Approach: In this study, firstly, the light coupling model was introduced. Then light beam propagation mode after adhesive with the same refractive index as that of light path was presented and the function modeling of couple model was derived. Finally, a slantedplane structure of interface was considered and coupling approximation after adhesive was calculated. Results: Simulations and experimental results indicate that theoretical coupling efficiency and measured coupling efficiency agree with very well and the couple loss decrease about $0.4 \mathrm{~dB}$ after adhesive. Conclusion: The matching adhesive could obviously increase the coupling efficiency and the approximation function could realize to predict the variation of coupling efficiency before or after adhesive accurately.
\end{abstract}

Key words: Coupling efficiency, waveguide packaging, UV epoxy adhesive, single mode fiber, Gaussian beam

\section{INTRODUCTION}

The current trend in optics communication is developing to achieve greater system performance, larger capacity and more fixable form. Advanced optoelectronic devices and new system with greater communication performance, such as higher data rates, smaller power dissipation or higher functionality integration, become prerequisites of the future optical communication. Samples of these devices are numerous, for example, Mems optical switches and tunable optical branching filters (Rahman et al., 2009; Ab-Rahman et al., 2009). Next generation optical communication system, namely all optics network, supporting terabit/sec data rate, will be developed with Dense Wavelength Division Multiplexing (DWDM), electro-optic modulators, photonic integrated circuits (Bchir et al., 2010), low-loss heterostructure waveguides and multi-wavelength quantum dot laser arrays (Zhang and Shi, 2004). In DWDM systems, some nanostructures are frequently used for optical signal processing, which include VCSEL lasers, all- optical buffers and micro-resonators, In addition, there are some late model devices to emerge in the optical display and optical sensor fields, including holographic polymer dispersed liquid crystals and photonic crystals.

In order to realize an integrated optical system or high-performance application, alignment in multidomain, packaging and assembly of those nanostructures are required. Packaging, however, is a challenging problem for those devices are connected together by fiber arrays or other type of waveguides with sub-micron alignment accuracies. Now, the packaging manufactures of these components with manual alignment are typically labor intensive, slow and the devices with poor performance are made possibly. Even with the recent developments of devices and Manufacturing Technology, the packaging and assembly of optoelectronic devices remain as the possible critical difficulty to commercial application (Fukushima et al., 2009; Zhang et al., 2005).

Package and assembly automation is the key to ensure performance, stability and high consistency, while getting high yield, low cost. There are growing 
interests in the development of these automation techniques to improve photonic alignment and packaging efficiency and the researches and product developments have been performed and got many promising results at Newport, Polytech PI, Palomar and Suruga. Those techniques mainly base on a combination of machine vision, accuracy motion stages with nanometer resolution capacity and maximizing power alignments algorithm and realize highperformance alignment and complete assembly. In contrast with semiconductor industry, the optoelectronics manufacture cannot directly use the automation that achieves huge success in the semiconductor community since those automation equipments, in which the optical and geometric axes are often not coherent, do not suit in optoelectronic packaging and still remain low yield. As a result, packaging costs currently accounts for $60-80 \%$ of the entire photonic component costs (Jeonga et al., 2007).

Equipment with six degrees of freedom to adjust single mode fibers, planar waveguide and other optical modulator is usually required. Once the adjustment is completed, the optical components must be held together by adhesive, solder, or welder attachment techniques. As its flexibility, easy-handle and high reliability, adhesive gluing (such as epoxy) is widely used in 90\% photonic device packaging (Pliska and Bosshard, 2007). After adhesive gluing, how to estimate the coupling efficiency accurately is becoming of great importance. When some adhesive is positioned in light path, Refractive Index (RI) of the path will change the optical beam propagation condition, which brings about increase of Coupling Efficiency (CE) or decrease, but little research on the misalignment pattern after epoxy adhesive is reported, including alignment tolerances of coupling after adhesive gluing.

The model of coupling efficiency between a singlemode fiber and a laser diode or a fiber and a fiber is usually derived by the Gaussian beam approximation. However, when some epoxy is positioned in light path, changes of refractive index of the path, which produces attenuation of coupling efficiencies possibly, alter the optical beam propagation. So the second adjusting of alignment must be followed. Coupling efficiencies cannot be directly compared with each other of examples, owing to the differences of components, such as the different laser diodes, fibers, or PLC.

In this study, we first present near mode field distribution of light beam outgoing from a fiber end and the $\mathrm{CE}$ equation derived from the Gaussian beam approximation. Next we discuss rules of RI effecting on the Gaussian optical distribution and develop a coupling efficiency equation based on RI affects. An adhesive with the same refractive index as that of fiber core is most appropriate for couple efficiency owing to reducing the misalignment loss and minimizing the reflection loss. In the optimum coupling condition, alignment tolerances after adhesive gluing are calculated theoretically. The results of the simulations in this study indicated that more than $0.4 \mathrm{~dB}$ coupling efficiency has been improved.

\section{METERIALS AND METHODS}

Materials: The simulation and experiment have been done with two single-mode fibers which have same parameters listed in Table 1 . The core diameter of the fiber was $8 \mu \mathrm{m}$. When the light wavelength was $1.31 \mu \mathrm{m}$ the mode field diameter was $8.9 \mu \mathrm{m}$. The end plane of the fiber was polished to $8^{\circ}$.

Packaging of planar light waveguides and adhesive gluing: A waveguide device exploited for some desired optical functionality must be properly packaged before it become a quality product. Of all the processes involved in packaging, alignment of optical fibers to waveguides is the most important but also the most difficult, which has become a bottleneck in the manufacture of photonic devices. The packaging involves two steps mainly: (1) aligning optically a fiber or fibers array to waveguide devices and (2) fixing the components with some adhesive to establish a permanent coupling mechanically. There are two methods of aligning a fiber to a waveguides device, namely active alignment and passive alignment. Although active alignment is a time-consuming and low efficiency adjustment, it makes advantage of the stateof-the-art technology based on the mature semiconductor automation, robotics, motion control, sensors and capital equipment and realizes sub-micron accuracy alignments easily. Consequently, active alignment is still the most main technology used in the manufacture of photonic devices.

After aligning a fiber to a waveguide, some kind of adhesive which refractive index is often the same as that of fiber core is dispensed in the gap between the fiber and the waveguide as shown in Fig. 1. The matching adhesive is used for two reasons practically: (1) attaching the components mechanically for life-time service; (2) improving the optical performance. The adhesive increases the refractive index of the light path, decreases simultaneously the reflective loss occurred on the reflective plane, and furthermore the adhesive redirects the light propagation for the end face of fiber array is slant. After adhesive gluing, second alignment is necessary. 
Am. J. Nanotech., 1 (2): 68-77, 2010

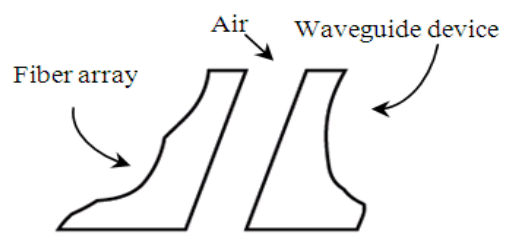

(a)

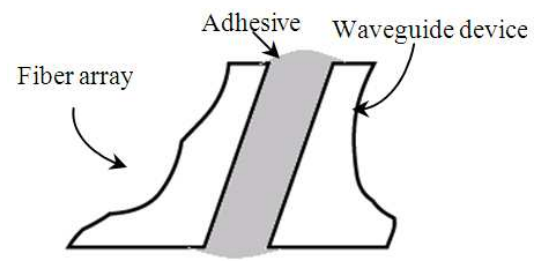

(b)

Fig. 1: The gap filled with air or adhesive,(a) air filled in the gap between fiber array and waveguide; (b) optical components attached by adhesive

Table 1: Single-mode fiber parameters

\begin{tabular}{ll}
\hline Items & Value \\
\hline Core diameter & $8 . \mu \mathrm{m}$ \\
Clad diameter & $125 \mu \mathrm{m}$ \\
Core refractive index & 1.45 \\
$\lambda_{\mathrm{c}}$ & $1.279 \mu \mathrm{m}$ \\
$\Delta$ & $0.3 \%$ \\
V number & $2.360($ at $1.31 \mu \mathrm{m})$ \\
N.A. & 0.123 \\
$\mathrm{~W}_{0}$ & 4.45 \\
\hline
\end{tabular}

Gaussian beam propogation and theoretical calculation of coupling efficiency: We describe the Gaussian beam outgoing from fiber end and the coupling characteristic between a single-mode fiber and waveguide. In order to calculate the coupling efficiency and alignment tolerance, the Gaussian approximation is adopted, which is sufficient to calculate coupling characteristics (Saruwatari and Nawata, 1979).

Near mode field of single-mode fibers: The power distribution of a single-mode fiber can be approximated by a Gaussian beam with $1 \%$ error. On the weak guidance condition, vectors $\mathrm{Ez}$ and $\mathrm{Hz}$ only along $\mathrm{z}$ are independent and they satisfy the scalar helmholtz equations in the cylindrical coordinate system Eq. 1:

$\nabla_{\mathrm{t}}^{2} \mathrm{~A}(\mathrm{r})+2 \mathrm{ik} \frac{\partial \mathrm{A}(\mathrm{r})}{\partial \mathrm{z}}=0$

Where:

$\mathbf{A}(\mathbf{r})=\mathbf{A}(\mathrm{r}, \mathrm{z})$

and:

$$
\nabla_{\mathrm{t}}=\frac{\partial^{2}}{\partial \mathrm{r}^{2}}+\frac{1}{\mathrm{r}} \frac{\partial}{\partial \mathrm{r}}+\frac{1}{\mathrm{r}^{2}} \frac{\partial^{2}}{\partial \theta^{2}}
$$

Considering electric field or magnetic field amplitude of the fundamental mode of a single mode fiber, the Gaussian solution is Eq. 2:

$$
A(r, z)=A_{0} \frac{w_{0}}{w(z)} e^{-r^{2} / w^{2}(z)}
$$

where, $\mathrm{w}_{0}$ is beam waist radius, $\mathrm{w}(\mathrm{z})$ presents spot size radius about $\mathrm{z}$ Eq. 3:

$$
\mathrm{w}(\mathrm{z})=\mathrm{w}_{0}\left[1+\left(\frac{\lambda \mathrm{z}}{\pi \mathrm{w}_{0}^{2}}\right)^{2}\right]^{1 / 2}
$$

Where:

$\lambda=\lambda_{0} / \mathrm{n}=$ Wavelength of light in some medium with refractive index $n$

$\lambda_{0}=$ Wavelength in vacuum

We can estimate the far field divergence angle of the Gaussian beam by the following Eq. 4:

$$
\theta_{\mathrm{d}}=\lim _{\mathrm{z} \rightarrow \infty} \frac{\mathrm{w}(\mathrm{z})}{\mathrm{z}}=\frac{\lambda}{\pi \mathrm{w}_{0}}
$$

The normalized frequency $\mathrm{V}$ is defined Eq. 5:

$$
\mathrm{V}=\mathrm{k}_{0} \mathrm{aN} \cdot \mathrm{A} \cdot=\frac{2 \pi \mathrm{a}}{\lambda_{0}} \sqrt{\left(\mathrm{n}_{1}^{2}-\mathrm{n}_{2}^{2}\right)}
$$

Where:

N.A. $=$ The number aperture of fiber

$\mathrm{a} \quad=$ The fiber core radius

If $1.9<\mathrm{V}<2.4$, the beam spot size $\mathrm{w}_{0}$ at $\mathrm{e}^{-1}$ amplitude is calculated by the empirical Eq. 6 (Saruwatari and Nawata, 1979):

$$
\mathrm{w}_{0} \approx \mathrm{a}\left(0.65+1.619 / \mathrm{V}^{3 / 2}+2.879 / \mathrm{V}^{6}\right)
$$

According to boundary limited conditions of Maxwell equations, where electric/magnetic field in tangential direction is continuous, $A_{z}(r)$ is invariable in the fiber end surface; the waist radius $\mathrm{w}_{0}$ of the Gaussian beam outgoing from the fiber surface is equal to the mode field radius of the fiber.

From Eq. 2, 3, 6, we can depict the distribution of the Gaussian beam propagation, as shown in Fig. 2.

Couple coefficient derivation: The couple efficiency between an incident beam and a single-mode fiber fundamental mode field distribution can be estimated through their overlap integral Eq. 7: 
Am. J. Nanotech., 1 (2): 68-77, 2010

$$
\eta=\frac{\left[\iint E_{i}(r, \theta) E_{f}^{*}(r, \theta) r d r d \theta\right]^{2}}{\left[\iint\left|E_{i}(r, \theta)\right|^{2} \operatorname{rdrd} \theta\right]\left[\iint\left|E_{f}(r, \theta)\right|^{2} \operatorname{rdrd} \theta\right]}
$$

Where:

$$
\begin{aligned}
\mathrm{E}_{\mathrm{i}}(\mathrm{r}, \theta), \mathrm{E}_{\mathrm{f}}(\mathrm{r}, \theta)= & \begin{array}{l}
\text { Denotes the lateral electric field } \\
\text { amplitude of the incident beam and } \\
\text { the coupled fiber, respectively }
\end{array} \\
\mathrm{CL} & =\text { Power coupling loss }
\end{aligned}
$$$$
\Gamma=-10 \lg [\eta](\mathrm{dB})
$$

The misalignments between an incident Gaussian beam and a single-mode fiber are shown in Fig. 3. The spot size radius of the incident beam is $\mathrm{w}_{\mathrm{i}}$ and that of the out fiber is $\mathrm{w}_{\mathrm{f}}$. The space between two fibers is denoted by z. The fiber axis tilts $\theta$ from the incident axis and dislocates $d_{r}$ from the incident axis. The coupling efficiency between two Gaussian beams is calculated Eq. 9 and 10 (Saruwatari and Nawata, 1979):

$$
\begin{gathered}
\eta=\mathrm{kExp}\left(-\mathrm{k}\left\{\frac{\mathrm{d}_{\mathrm{r}}^{2}}{2}\left(\frac{1}{\mathrm{w}_{\mathrm{i}, 0}^{2}}+\frac{1}{\mathrm{w}_{\mathrm{f}, 0}^{2}}\right)\right.\right. \\
\left.\left.+\frac{\pi^{2} \theta^{2}}{2 \lambda^{2}}\left[\mathrm{w}_{\mathrm{i}}^{2}(\mathrm{z})+\mathrm{w}_{\mathrm{f}, 0}^{2}\right]-\frac{\mathrm{d}_{\mathrm{r}} \theta \mathrm{z}}{\mathrm{w}_{\mathrm{i}, 0}^{2}}\right\}\right)
\end{gathered}
$$

Where:

$$
\begin{aligned}
& \mathrm{k}=\frac{4 \mathrm{w}_{\mathrm{i}, 0}^{2} \mathrm{w}_{\mathrm{f}, 0}^{2}}{\left(\mathrm{w}_{\mathrm{i}, 0}^{2}+\mathrm{w}_{\mathrm{f}, 0}^{2}\right)^{2}+(\lambda \mathrm{z} / \pi)^{2}} \\
& \mathrm{w}_{\mathrm{n}}^{2}(\mathrm{z})=\mathrm{w}_{\mathrm{n}, 0}^{2}\left[1+\left(\frac{\lambda \mathrm{z}}{\pi \mathrm{w}_{\mathrm{n}, 0}^{2}}\right)^{2}\right], \mathrm{n}=\mathrm{i}, \mathrm{f}
\end{aligned}
$$

And $w_{n, 0}$ means the mode field radius or Gaussian beam waist radius.

Near mode field of a slant-ended fiber: In the practical application, for reducing the return loss (Rahman et al., 2009; Luangpaiboon, 2011), a fiber is polished to an angle $\eta$, such as $\eta=5,8$, or 12 degree. The incident light beam detaches to two beams on the slant-end plane, including a reflective light beam and a transmitted light beam which is coupled to next fiber or waveguide. According to Fresnel reflective law, the electric vector of the incident light always decomposes to $\mathrm{TE}$ wave $\mathrm{E}_{\perp}$ which is perpendicular to the incident plane and $\mathrm{TM}$ wave $\mathrm{E}_{/ /}$parallel to that plane by contrast. For the light power dispersion relates with polarization of light, we investigate how two electric vectors transmit on the fiber end plane individually.

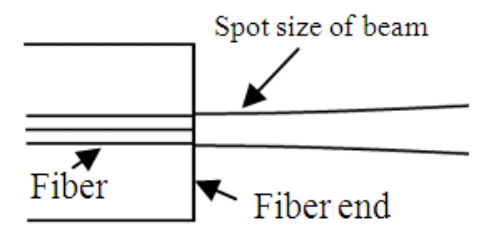

(a)

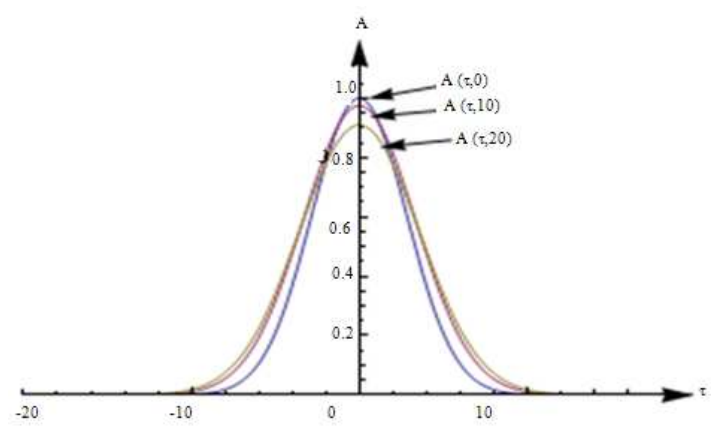

(b)

Fig. 2: Gaussian distribution of light outgoing from fiber end surface,(a) the spot size distribution from fiber end surface; (b) Gaussian mode field of $\mathrm{z}=0,10,20$,respectively

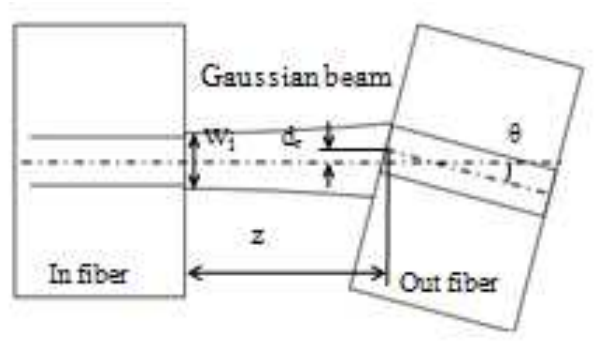

Fig. 3: Misalignment of two single-mode fibers

As the Gaussian light beam propagates along with the central axis of un-model fiber, the wave vector is parallel to the central axis as well. According to the unmode fiber light propagation direction, when the slant angle of the fiber end face is $\eta$ and the incident angle $\theta_{1}$, there is $\eta=\theta_{1}$.

About the TE wave transmission, according to Maxwell equations and boundary continuity condition, the relationships between the refractive electric vector and the reflective electric vector are built, as follows Eq. 11 and 12:

$$
\begin{aligned}
& \mathrm{E}_{\perp}^{\mathrm{r}}=\frac{\mathrm{n}_{1} \cos \theta_{1}-\mathrm{n}_{2} \cos \theta_{2}}{\mathrm{n}_{1} \cos \theta_{1}+\mathrm{n}_{2} \cos \theta_{2}} \bullet \mathrm{E}_{\perp}^{\mathrm{i}} \\
& \mathrm{E}_{\perp}^{\mathrm{t}}=\frac{2 \mathrm{n}_{1} \cos \theta_{1}}{\mathrm{n}_{1} \cos \theta_{1}+\mathrm{n}_{2} \cos \theta_{2}} \bullet \mathrm{E}_{\perp}^{\mathrm{i}}
\end{aligned}
$$


where, vectors $E_{\perp}^{\mathrm{i}}, \mathrm{E}_{\perp}^{\mathrm{r}}, \mathrm{E}_{\perp}^{\mathrm{t}}$ present the electric vectors of an incident wave, a reflective wave and a transmitting wave individually and the refractive indexes of two transparent mediums are $\mathrm{n}_{1}, \mathrm{n}_{2}$. In Fig. 4, the incident angle is $\theta_{1}$ and the transmission angle is $\theta_{2}$, they are Eq. 13:

$$
\mathrm{n}_{1} \sin \theta_{1}=\mathrm{n}_{2} \sin \theta_{2}
$$

From above equations, the transmittance $t_{\perp}$ of $\mathrm{TE}$ electric vector is constructed Eq. 14:

$$
\mathrm{t}_{\perp}=\mathrm{E}_{\perp}^{\mathrm{t}} / \mathrm{E}_{\perp}^{\mathrm{i}}=\frac{2 \mathrm{n}_{1} \cos \theta_{1}}{\mathrm{n}_{1} \cos \theta_{1}+\mathrm{n}_{2} \cos \theta_{2}}=\frac{2 \sin \theta_{2} \cos \theta_{1}}{\sin \left(\theta_{1}+\theta_{2}\right)}
$$

where, the variables $E_{\perp}^{t}, E_{\perp}^{t}$ are the magnitude of the vector $\mathrm{E}_{\perp}^{\mathrm{t}}, \mathrm{E}_{\perp}^{\mathrm{t}}$.

By the same way, about TM wave, the electric vector $\mathrm{E}_{/ /}^{\mathrm{i}}, \mathrm{E}_{/ /}^{\mathrm{t}}$ and the transmittance $\mathrm{t}_{/ /}$is derived as well Eq. 15 and 16:

$$
\begin{aligned}
& \mathrm{E}_{/ /}^{\mathrm{t}}=\frac{2 \sin \theta_{2} \cos \theta_{1}}{\sin \left(\theta_{1}+\theta_{2}\right) \cos \left(\theta_{1}-\theta_{2}\right)} \cdot \mathrm{E}_{/ /}^{\mathrm{i}} \\
& \mathrm{t}_{/ /}=\mathrm{E}_{/ /}^{\mathrm{t}} / \mathrm{E}_{/ /}^{\mathrm{i}}=\frac{2 \sin \theta_{2} \cos \theta_{1}}{\sin \left(\theta_{1}+\theta_{2}\right) \cos \left(\theta_{1}-\theta_{2}\right)}
\end{aligned}
$$

\section{CE calculations after adhesive dispensed:}

Couple efficiency of two field modes after adhesive gluing: An entire device is linked mainly by three methods, such as epoxy gluing, laser welding or general soldering. Epoxy gluing is a favorite means to bond a passive photoelectron device together (Pliska and Bosshard, 2007). Compared to laser welding or soldering, the epoxy glue has a lot of merits, such as a variety of forms, flexible operations, adapting to various complex interface structure, yet without a premetal sleeve round a fiber.

In the package of passive optical devices, such as waveguide splitter and Wavelength Division Multiplexing (WDM), the epoxy with the RI similar to the core is dispensed in the light path. Owing to the Rayleigh-Sommerfeld diffraction in fiber cross-section, the Gaussian beam distribution dissipates slowly and the couple efficiency is improved.

If the epoxy is a medium with the refractive index $n_{m}$ and is dispensed in the space between the incident beam and the couple fiber, we rewrite the Eq. 3 as Eq. 17:

$$
\mathrm{w}_{\mathrm{n}}(\mathrm{z})=\mathrm{w}_{0}\left[1+\left(\frac{\lambda_{0} \mathrm{z}}{\pi \mathrm{n}_{\mathrm{m}} \mathrm{w}_{0}}\right)^{2}\right]^{1 / 2}=\mathrm{w}_{0}\left[1+\left(\frac{2 \mathrm{z}}{\mathrm{kw}_{0}}\right)^{2}\right]^{1 / 2}
$$

This denoted that the spot size as shown in Fig. 5a is changed for the RI $n_{m}$. When $z$ offset is fixed, the higher is refractive index, the larger becomes the spot size radius. If the waist radius of two light beams is equal, namely $\mathrm{w}_{\mathrm{i}, 0}=\mathrm{w}_{\mathrm{f}, 0}$, the couple efficiency equation is simplified as follows:

- Lateral offset $\mathrm{d}_{\mathrm{r}}$ only. The power couple loss is as same as that before epoxy Eq. 18:

$$
\Gamma_{\mathrm{r}}=4.343\left(\frac{\mathrm{d}_{\mathrm{r}}}{\mathrm{w}_{0}}\right)^{2}
$$

As Fig. 5b shows, the $\Gamma_{\mathrm{r}}$ do not decrease or increase, even though refractive index rises from 1.0-1.45.

- Longitudinal offset only, we obtain the couple loss Eq. 19:

$$
\begin{aligned}
& \Gamma_{\mathrm{z}}=-10 \lg \left[\frac{1}{1+\left(\mathrm{z} \lambda_{0} / 2 \pi \mathrm{n}_{\mathrm{m}} \mathrm{w}_{0}^{2}\right)^{2}}\right] \\
& =-10 \lg \left[\frac{1}{1+\left(\mathrm{z} \lambda_{0} / 2 \pi \mathrm{w}_{0}^{2}\right)^{2} /\left(\mathrm{n}_{\mathrm{m}}\right)^{2}}\right]
\end{aligned}
$$

As Fig. 5c describes, if refractive index rises from 1.0-1.45, the $\Gamma_{\mathrm{z}}$ decreases from $0.358-0.174 \mathrm{~dB}$ at $\mathrm{z}=30 \mu \mathrm{m}$ reversely.

- Angle alignment tilt $\theta$ only, the power couple loss rewrites as follows Eq. 20:

$$
\Gamma_{\theta}=4.343\left(\frac{2 \theta}{\theta_{\mathrm{d}}}\right)^{2}=171.44\left(\frac{\mathrm{w}_{0}}{\lambda_{0}}\right)^{2}\left(\theta \mathrm{n}_{\mathrm{m}}\right)^{2}
$$

This equation describes that the change $\Gamma_{\theta}$ coincides with $\mathrm{n}_{\mathrm{m}}^{2}$ and $\theta^{2}$, as shows in Fig. $5 \mathrm{~d}$.

\section{Redirection of a slant-ended fiber mode field after} adhesive: For decreasing the return loss, the end face of a fiber is polished to an angle. According to Fresnel reflecting law, when the refractive index of fiber core is unequal to that of the gap, the Gaussian light beam alters its origin direct, as drawn in Fig. 6.

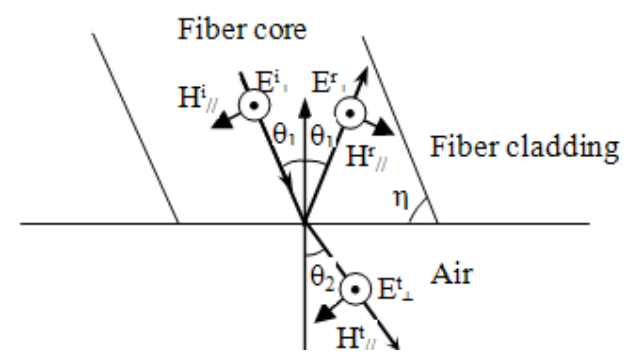

Fig. 4: TE wave transmission on slant-end plane of a fiber 


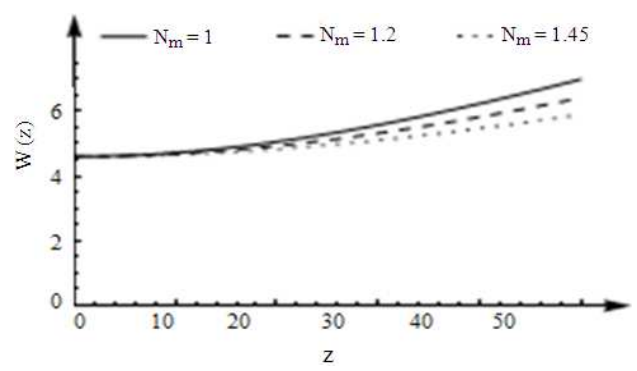

(a)

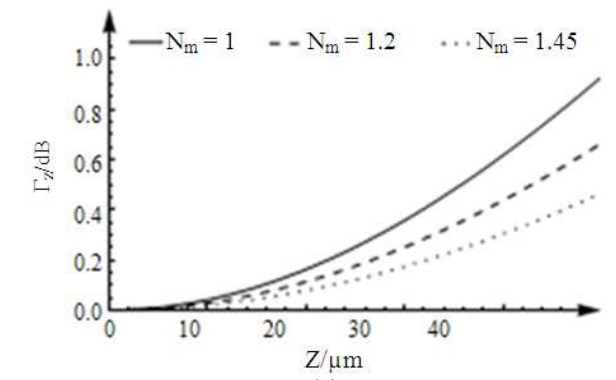

(c)

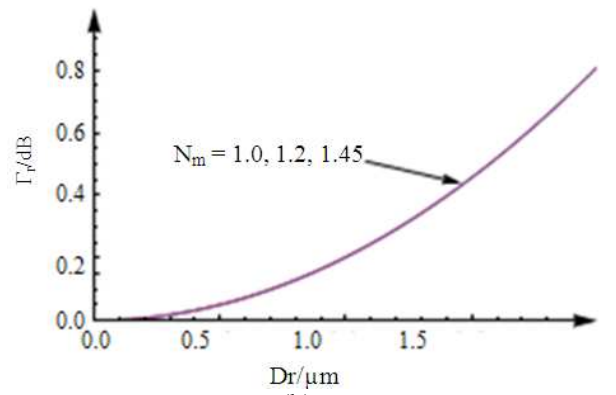

(b)

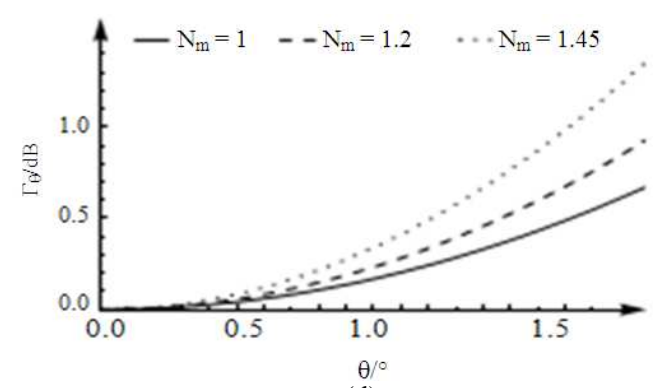

(d)

Fig. 5: Different RI effects on the spot size of Gaussian beam and the power coupling loss, (a) RI effects on the spot size of Gaussian beam; (b) $\Gamma_{\mathrm{r}}$ not affected by the RI; (c) $\Gamma_{\mathrm{z}}$ reducing with RI increasing; (d) $\Gamma_{\theta}$ rising with RI increasing

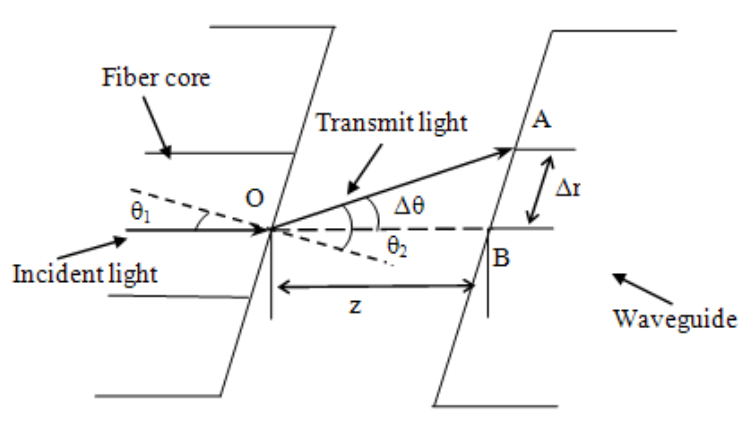

Fig. 6: Light redirecting for the slant-end face of a fiber.

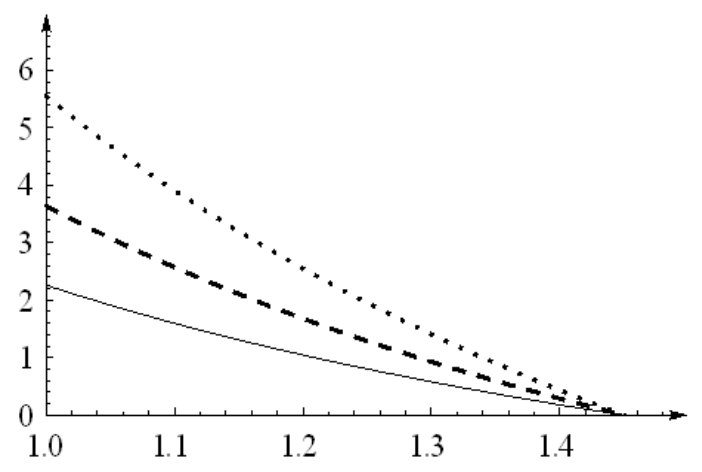

Fig. 7: $\Delta \theta$ According to the different RI and slant angle
$\mathrm{OA}$ is the transmitting light direction and $\mathrm{OB}$ follows the same direction of the incident light. If air is filled in the gap, or before adhesive gluing, the refractive index is about $\mathrm{n}_{2}=1.0$ and the transmitting angle is $\theta_{2}$. After an adhesive is dispensed in the gap, the light changes its direction from OA-OB.

According to Eq. 13, we can get the transmission angle $\theta_{2}$ Eq. 21:

$$
\theta_{2}=\arcsin \left(\frac{\mathrm{n}_{1}}{\mathrm{n}_{2}} \sin \theta_{1}\right)
$$

And then the difference of an incident angle and a transmission angle is got Eq. 22:

$$
\Delta \theta=\theta_{2}-\theta_{1}=\arcsin \left(\frac{\mathrm{n}_{1}}{\mathrm{n}_{2}} \sin \theta_{1}\right)-\theta_{1}
$$

Considering about these frequently-used slant angles $\left(\eta=5^{\circ}, 8^{\circ}, 12^{\circ}\right)$, the changing regularity of $\Delta \theta$ is shown in Fig. 7. When the refractive index $\mathrm{n}_{2}$ increases to that of fiber core 1.45(after adhesive gluing), the difference $\Delta \theta$ between the transmitting light and the incident light reduces to zero and the transmitting light keeps on transmitting in the direction of the incident 
light. Fig. 7 reveals that the more the fiber end face tilts, the farther the transmitting light deviates away from the incident light. For example, when the end face is polished to $5^{\circ}$, the difference $\Delta \theta$ is $2.26^{\circ}$ and when the angle tilts to $8^{\circ}, 12^{\circ}$, the $\Delta \theta$ increases from $3.64-5.55^{\circ}$ correspondingly.

After adhesive is injected in the gap, the change of the direction of the transmission light dislocates the coupling center from point A to B. For this reason, the model field alignment must be readjusted. In Fig. 7, the dislocation distance $\Delta \mathrm{r}$ is calculated Eq. 23:

$\Delta \mathrm{r} \approx \mathrm{ztg} \Delta \theta=\mathrm{ztg}\left(\arcsin \left(\frac{\mathrm{n}_{1}}{\mathrm{n}_{2}} \sin \theta_{1}\right)-\theta_{1}\right)$

where, $\mathrm{z}$ is the gap between a fiber to a fiber or a fiber array to a waveguide.

Fresnel transmission loss and power transmittance: As a light beam enters on the plane of two mediums, the power of incident light is redistributed to the reflective light and the transmission light on the assumption that the power is not absorbed and then a power redistribution expression is developed on Fresnel law. Considering the sectional area of a incident light $\Delta \mathrm{A}^{(\mathrm{i})}$ and energy flux density $S^{(i)}$, the energy flux is Eq. 24:

$\mathrm{W}^{(\mathrm{i})}=\mathrm{S}^{(\mathrm{i})} \Delta \mathrm{A}^{(\mathrm{i})}=\mathrm{S}^{(\mathrm{i})} \Delta \mathrm{A} \cos \theta_{1}$

where, $\theta_{1}$ is the slant angle between the sectional face and the reflective face and $\Delta \mathrm{A}$ is the corresponding area of $\Delta \mathrm{A}^{(\mathrm{i})}$ on the reflective plane. The light power of unit area every a second is Eq. 25:

$$
\mathrm{I}^{(\mathrm{i})}=\frac{\mathrm{S}^{(\mathrm{i})} \Delta \mathrm{A}^{(\mathrm{i})}}{\Delta \mathrm{A}}=\frac{\mathrm{S}^{(\mathrm{i})} \Delta \mathrm{A} \cos \theta_{1}}{\Delta \mathrm{A}}=\mathrm{S}^{(\mathrm{i})} \cos \theta_{1}
$$

The energy flux density $S^{(i)}$ is written as well Eq. 26:

$\mathrm{S}^{(\mathrm{i})}=\frac{1}{2} \sqrt{\frac{\varepsilon_{0}}{\mu_{0}}} \mathrm{n}_{1}\left|\mathrm{E}^{(\mathrm{i})}\right|^{2}$

Similarly, the transmission light power every a second is Eq. 27:

$$
I^{(t)}=\frac{S^{(t)} \Delta A^{(t)}}{\Delta A}==S^{(t)} \cos \theta_{2}
$$

And the energy flux density $\mathrm{S}^{(\mathrm{t})}$ of transmission light is Eq. 28:

$$
\mathrm{S}^{(\mathrm{t})}=\frac{1}{2} \sqrt{\frac{\varepsilon_{0}}{\mu_{0}}} \mathrm{n}_{2}\left|\mathrm{E}^{(\mathrm{t})}\right|^{2}
$$

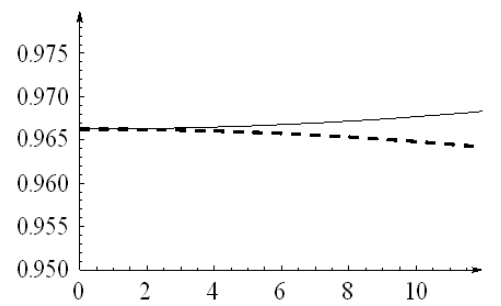

Fig. 8: Transmittance of the TE wave and the TM wave when incident angle is different

The light power transmittance is Eq. 29:

$\mathrm{T}=\frac{\mathrm{I}^{(\mathrm{t})}}{\mathrm{I}^{\mathrm{i}}}=\frac{\left|\mathrm{E}^{(\mathrm{t})}\right|^{2} \mathrm{n}_{2} \cos \theta_{2}}{\left|\mathrm{E}^{(\mathrm{i})}\right|^{2} \mathrm{n}_{1} \cos \theta_{1}}$

According to the Fresnel formula, the amplitude of electric vector relates to the polarization of light. For this reason, the electric vector $\mathrm{E}$ must be decomposed to $\mathrm{TE}$ wave $\mathrm{E}_{\perp}$ and $\mathrm{TM}$ wave $\mathrm{E}_{/ /}$. When the $\mathrm{E}$ vibration orientation and incident plane crosses at an angle, they are Eq. 30:

$\mathrm{E}=\mathrm{E}_{\perp} \cos \alpha+\mathrm{E}_{/ /} \sin \alpha$

Based on Eq. 11, 15 and 28, the transmittance of light propagation is established Eq. 31:

$\left\{\begin{array}{l}\mathrm{T}_{\perp}=\frac{\sin 2 \theta_{2} \cos 2 \theta_{1}}{\sin ^{2}\left(\theta_{1}+\theta_{2}\right)} \\ \mathrm{T}_{/ /}=\frac{\sin 2 \theta_{2} \cos 2 \theta_{1}}{\sin ^{2}\left(\theta_{1}+\theta_{2}\right) \cos ^{2}\left(\theta_{1}-\theta_{2}\right)}\end{array}\right.$

Substituted in the Eq. 8, the transmission loss is:

$\left\{\begin{array}{l}\Gamma_{\mathrm{T}_{\perp}}=-10 \lg \left(\mathrm{T}_{\perp}\right) \\ \Gamma_{\mathrm{T}_{/ /}}=-10 \lg \left(\mathrm{T}_{/ /}\right)=\Gamma_{\mathrm{T}_{\perp}}+10 \lg \left(\cos ^{2}\left(\theta_{1}-\theta_{2}\right)\right)\end{array}\right.$

According to Eq. 31, the different transmittance of TE wave and TM wave are drawn in Fig. 8.

When $\theta_{1}=0^{\circ}, 5^{\circ}, 8^{\circ}, 12^{\circ}$, the $\mathrm{T}_{/ /}$is $0.9662,0.9666$, $0.967,0.968$ and the $\mathrm{T}_{\perp}$ is $0.9662,0.9659,0.9653$, 0.9641. Although $\mathrm{T}_{/ /}$and $\mathrm{T}_{\perp}$ is different, this kind of difference is neglected in the practical application for simplifying calculation.

So:

$\mathrm{T} \approx \mathrm{T}_{\perp} \approx \mathrm{T}_{/ /}$

Equation 32 is rewritten in loss expression as follows Eq. 33:

$\Gamma_{\mathrm{T}} \approx \Gamma_{\mathrm{T}_{\perp}} \approx \Gamma_{\mathrm{T}_{/ /}}$ 


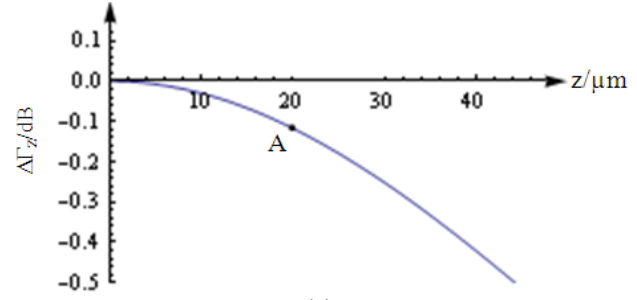

(a)

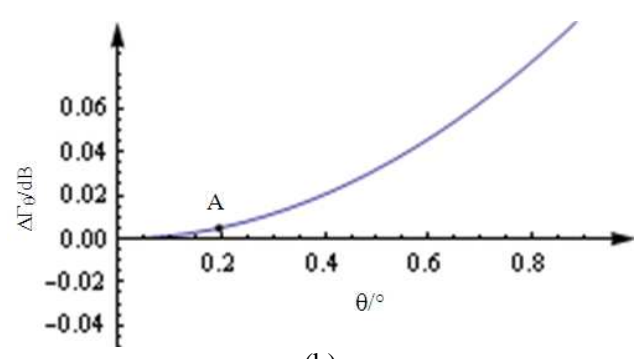

(b)

Fig. 9: Difference of the CL before and after epoxy. (a) CL affected by z-axis offset; (b) CL affected by angle tilt

Total power couple efficiency: In the optical alignment of photonic packaging, a light coupling from a fiber to a fiber or from a fiber to a waveguide is the most basic formation. A light outgoing from a fiber transmits on a fiber end face to a free medium and then continues to transmit into a fiber or a waveguide after a short excursion. There is a transmission loss in the conjunction planes of two medium for Fresnel reflection, except for the couple loss of misalignment. Thus the total coupling loss $\Gamma$ is Eq. 34:

$\Gamma_{\text {total }}=\Gamma+2 \mathrm{~T} \approx \Gamma_{\mathrm{r}}+\Gamma_{\mathrm{z}}+\Gamma_{\theta}+2 \Gamma_{\mathrm{T}}$

After adhesive is dispensed, the transmission loss reduces to zero and shrinking of spot size changes the couple loss.

The difference of the total coupling loss $\Gamma$ before adhesive gluing or after is Eq. 35:

$\Delta \Gamma_{\text {total }} \approx \Delta \Gamma_{\mathrm{r}}+\Delta \Gamma_{\mathrm{z}}+\Delta \Gamma_{\theta}+2 \Delta \Gamma_{\mathrm{T}}$

$=\Delta \Gamma_{\mathrm{z}}+\Delta \Gamma_{\theta}+2 \Delta \Gamma_{\mathrm{T}}$

\section{Simulations and Experiments:}

Coupling efficiency calculation: To specific effects of the epoxy in the opto-electronic packaging and assembly, we show a preliminary calculation about the power couple loss before epoxy and after epoxy Fig. 9. We provide two kinds of epoxy with refractive index of $\mathrm{n}_{\mathrm{m}}=1.2$ and $\mathrm{n}_{\mathrm{m}}=1.45$ compared to air $\mathrm{nm}=1.0$. If the excess power couple loss of a single channel limits to
$0.15 \mathrm{~dB}$, for example, we estimate the power couple loss before and after epoxy according to the Eq. 1820. After epoxy dispensing, the lateral dislocation tolerance $\mathrm{d}_{\mathrm{r}}$ restricts to $1.2 \mu \mathrm{m}$ as similar as that without epoxy; angle tilts tolerance $\theta$ decreases to $0.80^{\circ}$ and $0.67^{\circ}$ from $0.97^{\circ}$; Z-axis tolerance lengthen to 22.8 and 27.6 from $19.0 \mu \mathrm{m}$. Therefore, when the RI of epoxy is similar to that of the fiber core, it does not change the lateral dislocation tolerance, but reduce the angle inclination tolerance and increase the z-axial tolerance reversely.

We need to know how much the total change of the power coupling loss is improved after dispensing a certain epoxy. For example, we calculate fiber-to-fiber coupling loss, which parameters are listed in Table 1. If the refractive index of the epoxy is $\mathrm{n}_{\mathrm{m}}=1.45$, the difference of power coupling loss is described approximately as Eq. 36 and 37:

$\Delta \Gamma_{\mathrm{z}}=-10 \lg \left[\frac{1+\left(\mathrm{z} \lambda_{0} / 2 \pi \mathrm{n}_{0} \mathrm{w}_{0}^{2}\right)^{2}}{1+\left(\mathrm{z} \lambda_{0} / 2 \pi \mathrm{n}_{\mathrm{m}} \mathrm{w}_{0}^{2}\right)^{2}}\right]$

$\Delta \Gamma_{\theta}=4.343\left(\frac{2 \theta}{\lambda} \pi \mathrm{w}_{0}\right)^{2}\left(\mathrm{n}_{\mathrm{m}}-\mathrm{n}_{0}\right)^{2}$

About one point marked A as shown in Fig. 4, which $\mathrm{z}=20 \mu \mathrm{m}$ and tilts $0.2^{\circ}$, for example, we get:

$\Delta \Gamma_{\mathrm{z}}=-0.135(\mathrm{~dB}), \Delta \Gamma_{\theta}=0.008(\mathrm{~dB}) \approx 0 \mathrm{~dB}$

where, $\lambda_{0}=1.55 \mu \mathrm{m}$.

In addition, there are double Fresnel reflections. For a single-mode fiber listed in the Table 1 with contacted planes tilting $8^{\circ}$, we calculate difference of two power transmission rate:

$\Delta \Gamma_{\mathrm{t}}=\Gamma_{\mathrm{t}, \mathrm{n}_{2}}-\Gamma_{\mathrm{t}, \mathrm{n}_{1}} \approx-0.16(\mathrm{~dB})$ and $2 \Delta \Gamma_{\mathrm{t}}=-0.32 \mathrm{~dB}$

Consequently, the total coupling loss is:

$\Delta \Gamma=-0.135-0.32 \approx-0.46(\mathrm{~dB})$

Packaging experiments: In order to study effects of adhesive on the optical performance in practical photonic packaging, we used a fiber alignment system which designed by ourselves with sub-micron precision for the alignment of optical fibers to planar waveguides, The system was equipped with two six-axis nanopositioning stages and a power meter (Model 2835-C) from Newport (Bhat et al., 2004). On the two nanopositioning stages, we installed fiber-array holders. 


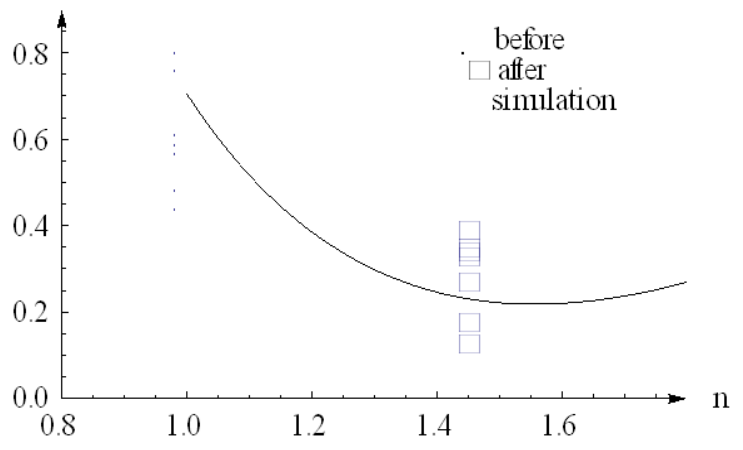

Fig. 10: Comparison between the couple simulation and the couple loss measured before and after adhesive when the slant angle $8^{\circ}$ of fiber, $\mathrm{z}$ offsets $<25 \mu \mathrm{m}$ and $\theta<0.2^{\circ}$, besides transverse offset $<0.4 \mu \mathrm{m}$

Firstly, two fibers which is slant to $8^{\circ}$ was installed on the holders, then we aligned automatically the fibers and measured the couple efficiency, at last, we realigned the fibers and measure the couple efficiency again after adhesive is dispensed. The measure results are shown in Fig. 10. The solid line described the simulation result that was compared with measure results before adhesive and after adhesive. The average difference of coupling loss was $0.4 \mathrm{~dB}$.

\section{RESULTS AND DISCUSSION}

In order to verify tolerances such as the lateral, angular misalignment and the z-axis offset, the theoretical results after adhesive were calculated. Compared with results before adhesive, maximum lateral misalignment tolerance is found to be $1.2 \mu \mathrm{m}$, but the maximum angle inclination tolerance reduces to about $0.67^{\circ}$ from $0.97^{\circ}$ and the longest $\mathrm{z}$ axial offset lengthens from 19.0-27.6 $\mu \mathrm{m}$ when the excess loss limits to $0.15 \mathrm{~dB}$

We achieved to calculate the total power couple loss about position misalignment and surface reflection after adhesive. The total power coupling efficiency was improved about $0.4 \mathrm{~dB}$.

\section{CONCLUSION}

In this study, we investigated the effects of adhesive on the power coupling loss based on the Gaussian beam propagation method. When a kind of adhesive dispensed on the interface of fibers changed the light propagation, the $\mathrm{z}$-axis displacement tolerance increased rather than angle tolerance degraded. According to the Gaussian beam coupling mode, an approximation function was built to estimate changes of coupling efficiency before and after adhesive. For the slanted end plane of fiber change the beam propagation direction, realignment in transverse plane was needed after adhesive.

Comparison between the simulation and the experiments indicated that the theoretical values and measured ones agreed with very well and the matching adhesive obviously increased the coupling efficiency. According the approximation function, the variation of coupling efficiency could be predicted accurately after adhesive.

\section{ACKNOWLEDGEMENT}

The researchers would like to thank the National Natural Science Foundation of China (50735007) and National 863 plans projects (2007AA04Z344).

\section{REFERENCES}

Ab-Rahman, M.S., H. Guna, M.H. Harun, S.D. Zan and K. Jumari, 2009. Cost-effective fabrication of selfmade $1 \times 12$ polymer optical fiber-based optical splitters for automotive application. Am. J. Eng. Applied Sci., 2: 252-259. DOI: 10.3844/ajeassp.2009.252.259

Bchir, R., A. Bardaoui, M. Machhout, R. Chtourou and H. Ezzaouia, 2010. Design and optimization of a 1.55 $\mu \mathrm{m}$ waveguide Based on silicon planar photonic crystals. Am. J. Applied Sci., 7: 77-80. DOI: 10.3844/ajassp.2010.77.80

Bhat, S.K., T.P. Kurzweg and A. Guez, 2004. Advanced packaging automation for optoelectronic systems. Proceedings of the Lightwave Technologies in Instrumentation and Measurement Conference, Oct. 19-20, pp: 11-16. DOI: 10.1109/LTIMC.2004.1370981

Fukushima, T., Y. Handa and K. Miyahara, 2009. Design of optical coupling systems between twodimensional quasi-stadium laser diodes and singlemode optical fibers. Opt. Rev., 16: 540-547. DOI: 10.1007/s10043-009-0106-4

Jeonga, S.H., G.H. Kimb and K.R. Cha, 2007. A study on optical device alignment system using ultra precision multi-axis stage. J. Mater. Proc. Technol., 187: 65-68. DOI: 10.1016/J.JMATPROTEC.2006.11.165.

Luangpaiboon, P., 2011. Constrained response surface optimization for a laser beam welding process. J. Math. Stat., 7: 5-11. DOI: 10.3844/jmssp.2011.5.11 
Pliska, A.C. and C. Bosshard, 2007. Adhesive bonding of passive optical components. Micro-Opto-Elect. Mater. Struct.: Phys., Mech., Design, Reliability, Packag., 487-525. DOI: 10.1007/0-387-329897_39

Rahman, M.S.A., F.A. Aziz, N.A.A.M. Arif, S.D. Zan and S.M. Mustaza, 2009. The polishing of cuttingedge polymer-on-glass for pigtailing preparation. Am. J. Eng. Applied Sci., 2: 127-132. DOI: 10.3844/ajeassp.2009.127.132

Saruwatari, M. and K. Nawata, 1979. Semiconductor laser to single-mode fiber coupler. Applied Opt., 18: 1847-1856. DOI: 10.1364/AO.18.001847
Zhang, Z., J. Liu, P. Zhao, G.Z. Xiao and C.P. Grover, 2005. Active alignment of optical fibers to planar waveguides using a thermal-curing adhesive. J. Lightwave Technol., 23: 567-570. DOI: 10.1109/JLT.2004.841259

Zhang, R. and F.G. Shi, 2004. A novel algorithm for fiber-optic alignment automation. IEEE Trans. Adv. Packag., 27: 173-178. DOI: 10.1109/TADVP.2004.825434 